\title{
Developments in the field of HIV estimates: methods, parameters and trends
}

Karen Stanecki, ${ }^{1}$ Geoff P Garnett, ${ }^{2}$ Peter D Ghys ${ }^{1}$

In the welter of official statistics, many can naïvely assume that numbers are based on actual counts. However, because of the effort required to collect and collate often rare or hidden events in difficult circumstances this is often not the case and estimates are required. In the case of HIV, infections are often asymptomatic, symptoms often untreated and people living with HIV often undiagnosed and unreported. Thus, estimates of the numbers of infections and cases of disease have to be built from partial data the representativeness of which has to be considered. Over the course of the HIV pandemic a continuous process of updating and improving the quality of data, our understanding of that data and the tools used to analyse the data has been undertaken, led by the Joint United Nations Programme on HIV/AIDS (UNAIDS) and with input from many scientists and technical experts. This effort should increase our confidence in the estimates of numbers of HIV infections and AIDS deaths, which are generated in partnership with national epidemiologists. Along the way the methods developed have been published in the peer review literature to allow critical scrutiny and to share our improving understanding of HIV epidemiology. We believe that the continued reassessment of methods and the transparency of their use have been important in establishing the validity of HIV estimates and facilitating their use in advocacy.

HIV estimates are used to understand the burden of disease and death caused by the virus, to understand trends in incidence and the impact of interventions. The distribution of infections and

\footnotetext{
${ }^{1}$ Joint United Nations Programme on HIV/AIDS (UNAIDS), Geneva, Switzerland; ${ }^{2}$ HIV/AIDS and TB, Global Health, Bill and Melinda Gates Foundation, Seattle, Washington, USA

Correspondence to Dr Karen Stanecki, Joint United Nations Programme on HIV/AIDS (UNAIDS), Geneva, 1211, Switzerland;StaneckiK@unaids.org

UNAIDS Report 2012 Guest Editors

Karen Stanecki

Peter D Ghys

Geoff P Garnett

Catherine Mercer
}

numbers of people needing treatment should act as a guide to the global, national and local efforts to prevent and treat infections. HIV-associated mortality should show us when our treatment programmes are failing. Patterns of drug-resistant HIV are likewise indicative of how well treatment programmes are performing and also of problems building for the future. The source of new HIV infections is a sign of where our HIV prevention efforts are not yet adequate. However, in interpreting all these patterns we need to identify and understand the information from which our estimates are derived: HIV incidence estimates are derived from models fitted to HIV prevalence data, which provides better accuracy for historical rather than for current trends. Patterns of mortality are predicted from these trends, from death rates based on pretreatment observational cohorts and from estimates of treatment coverage and effectiveness: with effectiveness estimated from clinical cohorts. Predictions of the source of new infections are based on models of transmission with many assumptions of the contacts between populations and the risks of transmission. Understanding the methods used to generate the estimates should lead to a better appreciation of how robust different numbers are and how well they can guide policy planning, implementation and evaluation.

This collection of papers provides an update on HIV estimation, exploring a variety of methods and analyses of primary data. The collection is one of a series where papers have focused on the tools used in the current round of estimates (in this case the 2011 set), the data and parameter estimates applied, proposals for future developments, some results and other related topics.

The 2011 set of UNAIDS/WHO estimates builds on improvements in estimation methods and assumptions: Bao et al ${ }^{1}$ describe the flexible epidemiological model that allows HIV infection risk to vary over time. The force of infection parameter, $r$, is allowed to vary over time through a random walk formulation, which can represent a greater variety of epidemic shapes continuing beyond the simple epidemic curve used previously. This has the advantage of allowing a closer representation of the data and the new patterns emerging as HIV epidemics age in populations. An informative prior distribution is used to improve short-term projections beyond the last year of data. Stover et $a l^{2}$ describe the major changes in the software used by most countries. They include integrated software bringing the free-standing Estimation and Projection Package (EPP) and Spectrum together into a single tool, a flexible model to fit epidemic trajectories, regionspecific survival of people on antiretroviral therapy (ART), updated estimates of mother-to-child transmission rates and new procedures to estimate uncertainty ranges around regional estimates.

A new question after a decade of using national surveys as a gold standard, ${ }^{3}$ is whether these national surveys give us unbiased estimates of prevalence based on inferences about who does not take part in surveys. Hogan et al $l^{4}$ suggest CIs should be much wider and that Heckman type analyses of non-response related to interviewer identity, provide improved point estimates. However, further research seems needed before such analyses should inform alternative point estimates since they generate counter-intuitive results in some countries. For example, the female to male ratios derived for Côte d'Ivoire and Zambia are less than 1 while they are more than 1 in all other countries with this new method, with the ratio more than 4:1 in Ghana, almost double the largest female to male ratio observed in unadjusted data (that for Senegal). Furthermore the application of Heckman type methods would lead to an approximate doubling of observed prevalence in Ghanaian and Malian females and in Ivorian, Malian and Zambian males. It is unclear whether major changes in prevalence can safely be based on analyses using interviewer identity and more work is required on the statistical properties of these analyses, but it should add caution in the interpretation of results when people are missed in sampling from populations.

In exploring the size and risk of particular key groups, the length of time they stay at risk, and can be found in surveys, is important. The review of duration of high risk behaviour by Fazito et al describes how duration is different for different key populations, and how for some key population groups the duration differs by region. The information is programmatically important, as a short duration implies that 
programmes need to actively approach new entrants. It is also important for estimation as it demonstrates that prevalence among key population groups changes or remains stable because of HIV transmission dynamics and because of the demographic dynamics of the population at risk.

Yiannoutsos et $a l^{6}$ estimate mortality of adult HIV-infected patients starting antiretroviral therapy from cohorts in five regions: East, Southern and West Africa, Asia Pacific and Latin America. Age, gender and CD4 count at the initiation of therapy were factors considered as predictors of mortality at 6, 12, 24 and $>24$ months after the start of treatment. They conclude that despite advances in antiretroviral treatment coverage, many patients start treatment at very low CD4 counts and experience significant mortality during the first 6 months after treatment initiation.

Rollins et $a l^{7}$ describe the derivation of a new set of estimates of mother-to-child transmission risks, building on evidence available through 2011. An important insight is that breastfeeding-related transmission rates are quite high, which is of particular importance as currently the breastfeeding period appears to be less covered with prophylactic regimens compared with the perinatal period. ${ }^{8}$

In addition to the methods and parameters employed in the 2011 estimates, two papers explore methods that could improve future rounds, looking ahead to improvements in the underlying model that fits to the observations, with proposals for a modified spline-based model by Hogan et $a l^{9}$ and a refined random walk model by Le Bao. ${ }^{10}$

Exploring currently available data the paper by Mahy et $a l^{11}$ on trends in HIV prevalence among young people extends previous analyses showing that declining prevalence trends during the early part of the past decade ${ }^{12}$ continue through the start of the current decade.

The paper by Gouws et al ${ }^{13}$ gives an overview of results of the incidence by Modes of Transmission model in 25 countries during 2008-2012. While this approach has some shortcomings related to data availability, model structure and interpretation of results, bringing the results from countries in different regions together in a single paper does provide a good overview of transmission dynamics in different regions. The paper also makes clear that it may not be useful for programmatic purposes to try and identify a single 'driver' of the local epidemic, ${ }^{14}$ as programming should address a range of transmission modes and interventions. ${ }^{15}$
The paper by Fazito et al ${ }^{16}$ describes a retrospective analysis of registered deaths in the Brazilian Mortality System to quantify under-reporting of HIV/AIDS deaths and those misclassified to AIDS-related conditions in the 15-49 years old population in Brazil. Most HIV mortality estimates are based on model projections, but increasingly the use of alternative sources, such as registered deaths should help us assess the validity of the model estimates. Unlike many of the preceding papers, this paper by Fazito is focused on concentrated epidemics. HIV estimates in these concentrated epidemics are greatly hampered by the difficulties of identifying the size of key populations. This makes the use of corroborative data all the more important and has also led to an exploration of new methods such as those looked at by Weir et al ${ }^{17}$ which suggest the need for caution when interpreting data from prevalence and behaviour surveys among key populations. In their study from Liuzhou, China, they found different results when different sampling methods were used for sampling sex workers in the same broad geographical entity. For the purpose of national estimates, the study serves as a reminder that individual data points need to be carefully examined as to whether they are comparable with data on the same population group at an earlier time point-if they are not, they should not be used to construct a prevalence time trend.

Over three decades the collaborative work in developing and refining methods for estimation has provided great insight into the pandemic. We believe that the willingness to criticise past results and be open about using new approaches has enabled improvements and should be a model for other disease estimation processes. However, the challenges inherent in accurately tracking the HIV pandemic are great and further work is needed to improve and update estimates.

Funding None.

Competing interests None.

Provenance and peer review Commissioned; internally peer reviewed.

Accepted 26 October 2012

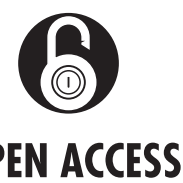

Sex Transm Infect 2012;88:1-2.

doi:10.1136/sextrans-2012-050885

\section{REFERENCES}

1. Bao L, Salomon J, Brown T, et al. Modeling HIV/AIDS epidemics in the UNAIDS Estimation and Projection Package 2011. Sex Transm Infect 2012;88 (Suppl 1):i3-i10.

2. Stover J, Brown T, Marston M. Updates to the Spectrum/EPP model to estimate HIV trends for adults and children. Sex Transm Infect 2012 88(Suppl 1):i11-i16.

3. Boerma JT, Ghys PD, Walker N. Estimates of HIV-1 prevalence from national population-based surveys as a new gold standard. Lancet 2003;362:1929-31.

4. Hogan D, Salomon J, Canning D, et al. National HIV prevalence estimates for sub-Saharan Africa: controlling selection bias with Heckman-type selection models. Sex Transm Infect 2012; 88(Suppl 1):i17-i23.

5. Fazito $\mathbf{E}$, Cuchi P, Mahy M, et al. Analysis of duration of risk behavior for key populations: a literature review. Sex Transm Infect 2012; 88(Suppl 1):i24-i32.

6. Yiannoutsos CT, Johnson L, Boulle A, et al. Estimated mortality of adult HIV-infected patients starting treatment with combination antiretroviral therapy. Sex Transm Infect 2012;88(Suppl 1): i33-i43.

7. Rollins N, Mahy M, Becquet R, et al. Estimates of peripartum and postnatal mother-to-child transmission probabilities of HIV for use in Spectrum and other population-based models. Sex Transm Infect 2012;88(Suppl 1):i44-i51.

8. UNAIDS. Toghether we will end AIDS. 2012.

9. Hogan D, Salomon J. Spline based modeling of trends $\mathrm{n}$ the force of HIV infection, with application to the UNAIDS EStimation and Projection Package. Sex Transm Infect 2012;88(Suppl 1):i52-i57.

10. Bao L. A new infectious disease model for estimating and projecting HIV/AIDS epidemics. Sex Transm Infect 2012;88(Suppl 1):i58-i64.

11. Mahy M, Garcia-Calleja JM, Marsh K. Trends in HIV prevalence among young people in generalized epidemics: implications for monitoring the HIV epidemic. Sex Transm Infect 2012;88(Suppl 1): i65-i75.

12. The International Group on Analysis of Trends in HIV Prevalence and Behaviours in Young People in Countries most Affected by HIV. Trends in HIV prevalence and sexual behaviour among young people aged 15-24 years in countries most affected by HIV. Sex Transm Infect 2010;86 (Suppl 2):ii72-83.

13. Gouws E, Cuchi P. Focusing the HIV response through estimating the major modes of HIV transmission: a mulit-country analysis. Sex Transm Infect 2012;88(Suppl 1):i76-i85.

14. Mishra S, Sgaier SK, Thomson L, et al. HIV epidemic appraisals for assisting in the design of effective prevention programmes: shifting the paradigm back to basics. PLOS ONE 2012;7.

15. Schwartlander B, Stover J, Hallett T, et al. Towards an improved investment approach for an effective response to HIV/AIDS. Lancet 2011;377:2031-2041.

16. Fazito $\mathbf{E}$, Cuchi P, Fat D, et al. Identifying and quantifying misclassified and under-repported AIDS deaths in Brazil: a retrospective analysis from 1983 to 2009. Sex Transm Infect 2012;88(Suppl 1): i86-i94.

17. Weir SM, Merli MG, Li J, et al. A comparison of respondent-driven and venue-based sampling of female sex workers in Liuzhou, China. Sex Transm Infect 2012;88(Suppl 1):i95-i101. 